\title{
Effects of trimetazidine on ventricular remodeling in coronary artery disease patients with left ventricular hypertrophy: the rationale and design of a randomized controlled trial
}

\author{
Lili Wu', Yi Luan ${ }^{\dagger}, \mathrm{Ya} \mathrm{Li}^{\dagger}$, Min Wang, Jialin He, Chongying Jin and Wenbin Zhang ${ }^{*}$ (i)
}

\begin{abstract}
Background: Trimetazidine is a metabolic anti-ischemic agent, which increases the tolerance of cardiomyocytes to ischemia. However, few studies have explored the effect of trimetazidine on ventricular remodeling in coronary artery disease (CAD) patients undergoing percutaneous coronary intervention $(\mathrm{PCl})$ with left ventricular hypertrophy $(\mathrm{LVH})$.

Methods: It is a randomized, placebo-controlled trial, and we propose to recruit one hundred and twenty-four CAD patients undergoing $\mathrm{PCl}$ with $\mathrm{LVH}$ during a 12-month period. They will be randomized to receive either trimetazidine (35 mg twice a day) or placebo in the following 12 months after $\mathrm{PCl}$. Blood tests, echocardiography, symptom of angina and major adverse cardiovascular events (MACEs) will be collected at follow-up visit at 3 and 12 months. The primary end point will be the left ventricular remodeling measured by left ventricular mass index (LVMI) at 3- and 12-month follow-up compared with the baseline. The secondary end points will be the symptom of angina assessed by Seattle Angina Questionnaire, myocardial ischemia measured by 6-min walk test and exercise electrocardiography test, as well as MACEs (defined as a composite of death, myocardial infarction, stroke, recurrent angina, re-hospitalization, change of viable myocardium).
\end{abstract}

Discussion: This study aims to demonstrate the effect of trimetazidine on left ventricular remodeling and myocardial ischemia in CAD patients undergoing PCI with LVH. Trimetazidine treatment is likely to improve the left ventricular remodeling, symptoms of angina and myocardial ischemia. It might also reduce the risk of MACEs in CAD patients undergoing $\mathrm{PCI}$ with $\mathrm{LVH}$.

Trial registration: http://www.chictr.org.cn, Chinese Clinical Trial Registry (ChiCTR1800017876). Registered on 19 Aug 2018.

Keywords: Trimetazidine, Ventricular remodeling, Myocardial ischemia

\footnotetext{
* Correspondence: 3313011@zju.edu.cn

${ }^{+}$Lili Wu, Yi Luan and Ya Li contributed equally to this work. Department of Cardiology, Key Laboratory of Cardiovascular Intervention and Regenerative Medicine of Zhejiang Province, Sir Run Run Shaw Hospital, School of Medicine, Zhejiang University, 3 East Qingchun Road, Hangzhou, Zhejiang 310016, P.R. China
}

C C The Author(s). 2020 Open Access This article is licensed under a Creative Commons Attribution 4.0 International License, which permits use, sharing, adaptation, distribution and reproduction in any medium or format, as long as you give appropriate credit to the original author(s) and the source, provide a link to the Creative Commons licence, and indicate if changes were made. The images or other third party material in this article are included in the article's Creative Commons licence, unless indicated otherwise in a credit line to the material. If material is not included in the article's Creative Commons licence and your intended use is not permitted by statutory regulation or exceeds the permitted use, you will need to obtain permission directly from the copyright holder. To view a copy of this licence, visit http://creativecommons.org/licenses/by/4.0/. The Creative Commons Public Domain Dedication waiver (http://creativecommons.org/publicdomain/zero/1.0/) applies to the data made available in this article, unless otherwise stated in a credit line to the data. 


\section{Background}

Coronary artery disease (CAD) has become the second leading cause of death after malignant tumor in China, with the prevalence of $6.46 \%[1,2]$. Left ventricular hypertrophy $(\mathrm{LVH})$, an increase in muscle mass of left ventricle, has been identified as a powerful risk factor for future cardiovascular morbidity and mortality, with the risk of cardiovascular events increasing six-fold to eightfold [3]. In addition, LVH is independently associated with a higher risk of all-cause mortality and a higher incidence of heart failure in CAD patients treated with percutaneous coronary intervention (PCI) [4-6]. LVH can develop myocytes death and fibrotic remodeling, and this promotes cardiac dysfunction [7], leading to cardiac hypoxia [8].

Besides, microvascular dysfunction is a recognized feature of hypertrophic cardiomyopathy and it may exacerbate myocardial ischemia in CAD patients [9]. Although PCI has become one of the leading treatment methods for coronary heart diseases, microcirculation disturbance and cardiac metabolic disorders caused by LVH still cannot be effectively relieved. Distinctive from other anti-anginal agents, trimetazidine is an anti-ischemic metabolic agent [10-12]. Trimetazidine optimizes the energy utilization of myocardial cells and maintains a proper energy supply during ischemia [13]. It protects the heart by inhibiting oxidative stress [14], scavenging oxygen free radicals $[15,16]$, improving lipid metabolism and mitochondrial function [14, 17], and reducing ionic imbalance during ischemia and reperfusion [18]. Researchers have reported the protective effect of trimetazidine in CAD patients undergoing PCI $[19,20]$. However, its long-term effect on left ventricular remodeling in CAD patients undergoing PCI is still unclear.

This prospective randomized trial is therefore designed to explore whether trimetazidine can improve ventricular remodeling in CAD patients with $\mathrm{LVH}$, as well as trying to investigate whether trimetazidine treatment can reduce major adverse cardiovascular events (MACEs). This study is to provide evidence for further clinical application of trimetazidine in CAD patients undergoing PCI with LVH.

\section{Methods/design}

\section{Study design}

It is the first prospective, placebo-controlled study to explore the relationship between trimetazidine and ventricular remodeling. CAD patients undergoing PCI with $\mathrm{LVH}$ will be randomized to receive trimetazidine $(35 \mathrm{mg}$ twice a day) or placebo for 12 months after PCI. Coronary intervention and other medication therapy will be performed according to current guidelines, and no difference in the use of angiotensin-converting enzyme inhibitor (ACEI), angiotensin receptor blocker (ARB) and aldosterone antagonist should be ensured between the two groups. The web-based randomization will be used for a 1:1 ratio grouping to either trimetazidine or placebo. Secretary of department of cardiology accesses the website via account password and is responsible for randomization. Block randomization will be stratified by concomitant medications (ACEI, ARB or aldosterone antagonist), sex and family history and it is blind to those who enroll participants or assign interventions. Blood biochemistry, echocardiography, Seattle Angina Questionnaire, 6-min walk test (6-MWT), exercise electrocardiography test and MACEs will be collected at the 3- and 12-smonth clinical follow-ups. All personal information will be converted to the new subject number. The attending physicians are responsible for data entry. Data monitoring committee is responsible for data security and accuracy. Committee members (Fen $\mathrm{Xu}$, Jinshan Tong and Jiangfen Jiang) have access to the final dataset and periodically range check for data values.

The primary end point is the change of ventricular remodeling assessed by left ventricular mass index (LVMI) at 3- and 12-month follow-up after PCI compared with the baseline. The secondary end points will be the symptom of angina assessed by Seattle Angina Questionnaire, myocardial ischemia measured by 6-MWT and exercise electrocardiography test, as well as MACEs (defined as a composite of death, myocardial infarction, stroke, recurrent angina, re-hospitalization, change of viable myocardium). Details of the proposed study, including enrollment and follow-up visits, are outlined in Table 1. All serious adverse events must be reported to the sponsor immediately or within $24 \mathrm{~h}$ in case report forms (CRFs).

The trial protocol has been approved by the Institutional Ethics Committee of Sir Run Run Shaw Hospital of Zhejiang University (KY20180508-25) and has been registered in at Chinese Clinical Trial Registry (ChiCTR1800017876). All patients will provide written informed consents before randomization.

\section{Study objectives}

The trial has been designed to investigate the hypothesis that trimetazidine treatment $(35 \mathrm{mg}$ twice a day, for 12 months) could significantly improve the left ventricular remodeling, reduce the incidence of MACEs and effectively improve myocardial ischemia in CAD patients undergoing PCI with LVH. The primary objective is to validate that trimetazidine treatment could reduce LVMI and improve ventricular remodeling. The secondary objective is to validate that trimetazidine treatment could reduce the risk of MACEs, as well as improve the symptom of angina and myocardial ischemia. 
Table 1 Study plan

\begin{tabular}{|c|c|c|c|}
\hline \multirow[t]{2}{*}{ Activity } & Visit 0 screening & Visit 1 & Visit 2 \\
\hline & 0 day & 3 months \pm 7 day & 12 months \pm 7 day \\
\hline Inclusion/exclusion criteria & $x$ & & \\
\hline Informed consent & $x$ & & \\
\hline Randomization & $\times$ & & \\
\hline Demography and medical history & $x$ & & \\
\hline Physical examination & $\times$ & $\times$ & $\times$ \\
\hline 12-lead ECG & $x$ & $\times$ & $\times$ \\
\hline Concomitant medication & $\times$ & $\times$ & $\times$ \\
\hline CBC & $x$ & $x$ & $\times$ \\
\hline Scr, BUN, UA & $\times$ & $x$ & $x$ \\
\hline ALT, AST & $\times$ & $x$ & $x$ \\
\hline TC, LDL-C, HDL-C, TG & $\times$ & $x$ & $\times$ \\
\hline NT-proBNP & $\times$ & $x$ & $\times$ \\
\hline$c T n T / c T n l$ & $\times$ & & \\
\hline Echocardiography & $\times$ & $\times$ & $\times$ \\
\hline Symptoms of angina & $\times$ & $x$ & $\times$ \\
\hline 6 min walk test & $\times$ & $x$ & $\times$ \\
\hline Exercise electrocardiography test & $\times$ & $x$ & $\times$ \\
\hline MACES & & $\times$ & $\times$ \\
\hline Quality of life & $\times$ & $x$ & $x$ \\
\hline
\end{tabular}

ECG Electrocardiography, CBC complete blood count, Scr serum creatinine, BUN blood urea nitrogen, UA uric acid, $A L T$ alanine aminotransferase, $A S T$ aspartate aminotransferase, $T C$ total cholesterol, $L D L-C$ low-density lipoprotein cholesterol, HDL-C how-density lipoprotein cholesterol, $T G$ triglyceride, NT-proBNP N-terminal pro-B-type natriuretic peptide, $C T n T / C T n /$ cardiac troponin-T/ cardiac troponin-I, MACEs major adverse cardiovascular events

\section{Inclusion and exclusion criteria}

One hundred and twenty-four CAD patients undergoing PCI with LVH (LVMI > 120 and $100 \mathrm{~g} / \mathrm{m}^{2}$ for men and women, respectively) at Sir Run Run Shaw Hospital of Zhejiang University will be enrolled in the trial. The enrollment period is expected to be finished in 2020 . Detailed inclusion and exclusion criteria are listed in Table 2. The attending physician is responsible for the inclusion of patients. Patients treated with other medications which would affect cardiac metabolism, and patients who are concomitant with other causes of myocardial ischemia or other causes of LVH will be excluded from the study.

\section{Exclusion criteria during the follow-up periods}

Every participant will have the right to withdraw from the study without restriction, and participants may be removed if

1) Patients' demand to withdraw the informed consent.

2) Study protocol violation (including poor compliance).

3) Lost to follow-up.
4) Pregnancy or new-onset malignant tumor during the follow-up.

5) Detrimental to the patient's well-being.

Every withdrawal will be recorded in the CRFs, and a visit will be arranged if possible.

\section{Study procedures}

The study protocol is shown in Fig. 1 and Table 1. Patients are randomly allocated to receive trimetazidine (35 mg twice a day) or placebo for 12 months after PCI. All interventions are performed strictly comply with the standard. Physical examination, blood test, 12-lead electrocardiogram, echocardiography, symptoms of angina, 6-MWT, exercise electrocardiography test and quality of life will be collected at baseline visit and every follow-up visit. Blood tests will include complete blood count $(\mathrm{CBC})$, alanine aminotransferase (ALT), aspartate aminotransferase (AST), serum creatinine (Scr), blood ureanitrogen (BUN), low-density lipoprotein cholesterol (LDL-C) and N-terminal pro-B-type natriuretic peptide (NT-proBNP). MACEs will be collected at 3- and 12month follow-up. Research nurses will inform patients of the follow-up time in advance. They are helpful for 
Table 2 Inclusion and exclusion criteria

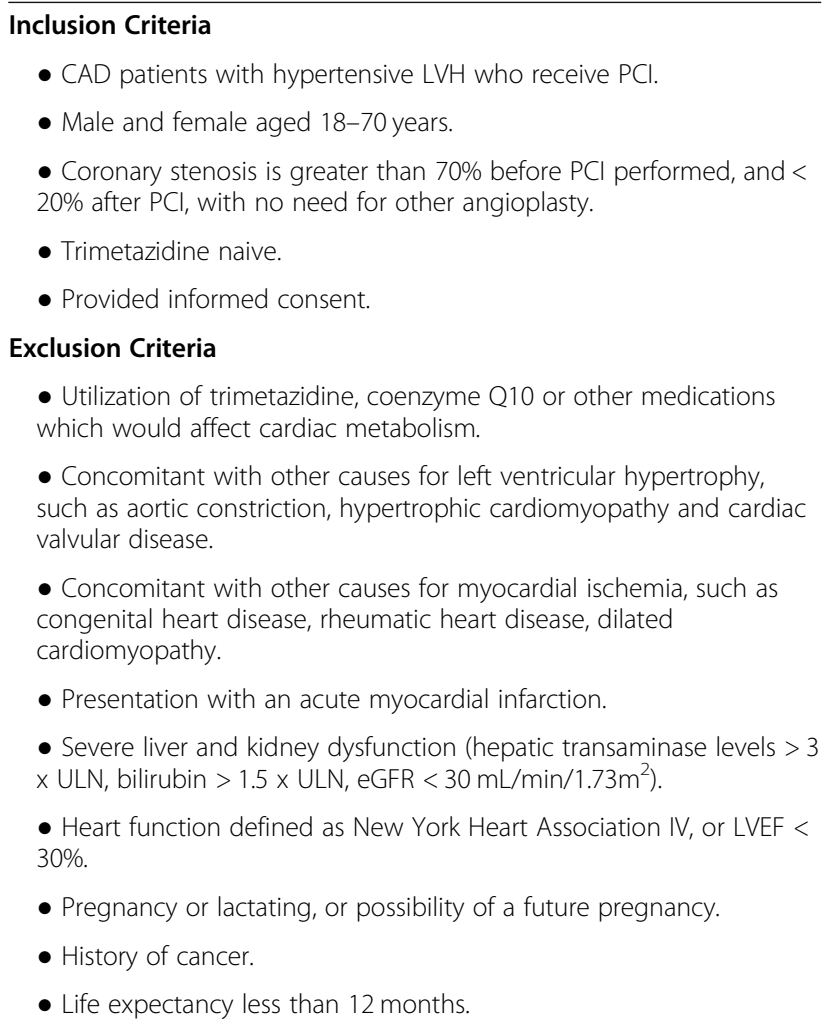

$C A D$ coronary artery disease, $L V H$ left ventricular hypertrophy, $P C I$ percutaneous coronary intervention ULN upper limit of normal range, eGFR estimated glomerular filtration rate, LVEF left ventricular ejection fraction completion of follow-up and monitoring adherence (e. g, drug tablet return).

\section{Primary end point}

The primary end point in this study is the change between baseline and follow-up LVMI (calculated as the ratio: left ventricular mass (LVM)/ body surface area). Two-dimensional and Doppler echocardiography will be performed to quantify left ventricular function and structure (Phillips Healthcare) [21]. Ventricular remodeling will be assessed by calculating LVMI using Devereux formula. Interventricular septum thickness (IVST), left ventricular end-diastolic dimension (LVEDd), left ventricular posterior wall thickness (LVPWT) will be measured by echocardiography. LVM = 1.04[(IVST + LVEDd $\left.+\mathrm{LVPWT}^{3}-\mathrm{LVEDd}^{3}\right]-13.6 \mathrm{~g}$.

\section{Secondary end points}

The secondary end points are the change the symptom of angina and myocardial ischemia, and the 3- and 12month incidence of MACEs. The symptom of angina will be assessed at baseline, 3- and 12-month follow-up period by Seattle Angina Questionnaire. The improvement of myocardial ischemia and exercise capacity will be measured using exercise electrocardiography test and 6-MWT. The 6-MWT is a sample, low-cost method for estimating exercise capacity and will be performed according to standard procedures [22]. Clinical characteristics and a multipurpose health survey that consists of 36 questions (short form-36, SF-36) will be recorded at baseline, 3 months and 12 months. MACEs are

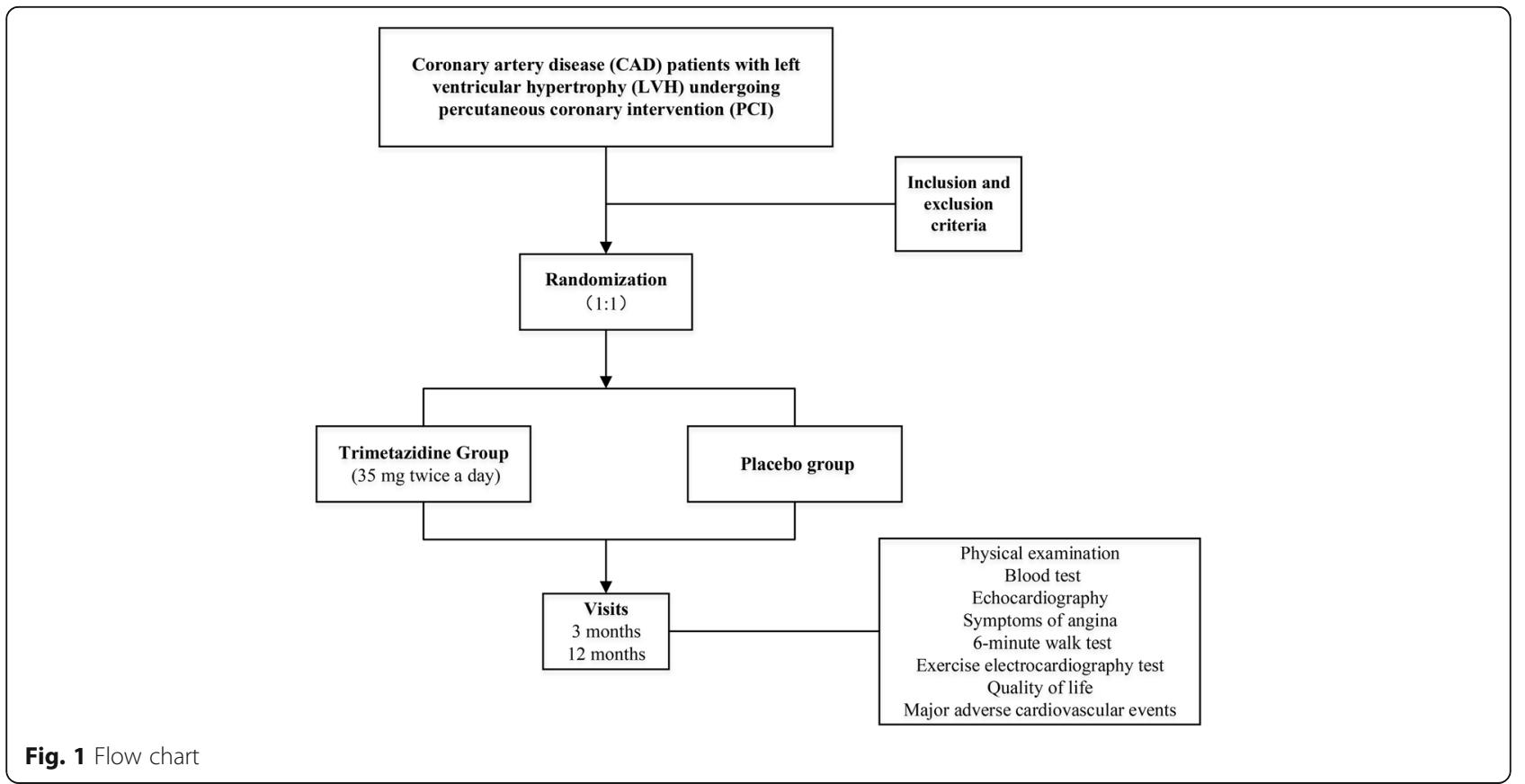


defined as a composite end point of death, myocardial infarction, stroke, recurrent angina, re-hospitalization, change of viable myocardium.

\section{Trial committees}

Wenbin Zhang guides the research team and provides overall supervision for the trial. The data monitoring committee (Fen $\mathrm{Xu}$, Jinshan Tong and Jiangfen Jiang) is responsible for reviewing and monitoring data safety. Investigators have the right to reveal a participant's allocated intervention or terminate the study in advance after discussion with Wenbin Zhang in the event of safety issues during the study as well as notify the ethics committee.

\section{Statistical considerations Sample size}

According to previous study [20], we hypothesized trimetazidine group has an effective rate of $70 \%$ to improve the left ventricular remodeling by reducing LVMI and placebo group has $40 \%$, thus 56 patients in each group are necessary to offer $90 \%$ power at 0.05 significant level according to 1:1 ratio of random grouping. Anticipating $10 \%$ of patients potentially non-assessable according to previous experience on 12-month study, 124 randomized patients in total are needed.

\section{Analysis}

The final results will be presented on an intention-totreat (ITT) basis. All data will be presented as percentages or mean \pm standard deviation (SD). The comparison of data will be performed using the Chi-squared test (categorical variables) or Student's $t$ test (continuous variables). Cumulative incidences of end points in 12 months after PCI will be calculated using Kaplan-Meier methods. Log-rank tests will be used for comparisons between groups. Possible covariables will be incorporated in the analysis if certain important baseline levels are still not matched after randomization. Mean imputation will be used to handle missing data. A $p$-value < 0.05 will be considered significant. All statistical analyses will be performed using the Statistical Package for Social Sciences (SPSS), version 20.0 (SPSS Inc., Chicago, IL, USA). The results will be published by chief investigator and major contributors.

\section{Discussion}

The study is the first investigator-initiated, prospective study to evaluate the treatment effect of trimetazidine on ventricular remodeling in CAD patients undergoing PCI with LVH. The result of the study will confirm whether trimetazidine improves ventricular remodeling, angina and myocardial ischemia.
Cardiac hypertrophy is a common pathological change in cardiovascular diseases. In the early stage, it is an adaptive response of the heart to the overload. With the development of the disease, the myocardial structure will be disordered and the compliance will be decreased. The mechanism of CAD associated with $\mathrm{LVH}$ is a complex process involving multiple factors, such as blood pressure, catecholamine and renin-angiotensin-aldosterone system. It is characterized by hypertrophy of cardiomyocytes and changes in coronary microcirculation. Coronary microcirculation networks are the metabolic site of myocardium, and their lesions can cause the decline of coronary reserve function and the changes of myocardial ultrastructure, which are closely related to the maintenance of myocardial energy metabolism.

Myocardial energy metabolism disorders occur prior to typical morphological changes in ventricular hypertrophy [23]. During the development of cardiac hypertrophy, the energy metabolism of hypertrophic cardiomyocytes changes from aerobic oxidation to anaerobic fermentation. Metabolic therapy is to improve the process of energy metabolism so that cardiomyocytes can get more energy. Trimetazidine is a metabolic agent with a protective effect on cardiomyocytes. It mainly inhibits the oxidation of free fatty acids, activates pyruvate dehydrogenase, increases the oxidation of glucose and the production efficiency of cardiac adenosine triphosphate (ATP), as well as maintains myocardial contractile function [24]. It also directs free fatty acids to synthesize phospholipids, which involve in the construction of cell membrane. Accordingly, by improving the environment of the cell and promoting the construction of membrane, trimetazidine might protect the ultrastructure of myocardium and is likely to inhibit or even reverse the cardiac hypertrophy.

Previous study has showed trimetazidine minimizes myocardial reperfusion injury during PCI and improves global and regional wall motion at one and 3 months after PCI [20]. In this previous study, 52 acute coronary syndrome (ACS) patients were included and randomized into trimetazidine group and placebo group. Echocardiographic measurements before revascularization revealed $40 \%$ patients in trimetazidine group had an ejection fraction $<50 \%$ versus $33 \%$ patients in placebo group. The number of patients with an ejection fraction $<50 \%$ was significantly reduced in trimetazidine group compared with placebo group after PCI (11\% vs. $16 \%, p=0.046$ at 1 month; and $4 \%$ vs. $16 \%, p=0.017$ at 3 months). A significant improvement in regional wall motion was noted after treatment with trimetazidine compared with placebo. Three months after PCI, inferior left ventricular wall hypokinesia had improved in $66.7 \%$ trimetazidine recipients and in $42.9 \%$ placebo recipients $(p=0.05)$, and anterior wall hypokinesia had improved in $72.7 \%$ in 
trimetazidine group and in $33.3 \%$ in placebo group ( $p=$ 0.04). However, the researchers only focused on the improvements for 3 months, long-term impact of trimetazidine on left ventricular remodeling in CAD patients undergoing PCI is still unclear. Subsequent Korean study suggested that trimetazidine treatment in non-ST segment elevation myocardial infarction (NSTEMI) patients provided improvements in left ventricular end diastolic volume [25]. The adjuvant treatment with trimetazidine after drug-eluting stent implantation was also confirmed to have a beneficial effect on left ventricular function and structure in elderly multivessel CAD patients with diabetes mellitus [26]. Nonetheless, these studies were not focused directly on patients with LVH. The study we proposed is intended to provide evidence for further clinical application of trimetazidine in CAD patients with LVH.

There were also studies in recent years exploring possible molecular mechanisms. The rat model of aortic constriction was established to induce myocardial metabolic disorder within 7 days, and it was found that trimetazidine pretreatment could improve the myocardial energy metabolism by reducing serum levels of oxidative stress markers, attenuating the induction of genes related to myocardial hypertrophy, inhibiting the upregulation of serum neuropeptide Y (NPY) levels, and further increasing the expression of myocardial NPY receptors [27]. This not only demonstrated that trimetazidine pretreatment could reverse acute metabolic disorders, but also revealed the underlying pathophysiological process of metabolic remodeling before the onset of myocardial remodeling.

Other researchers verified in vivo and in vitro that trimetazidine could effectively inhibit myocardial fibrosis through nicotinamide adenine dinucleotide phosphate $(\mathrm{NADPH})$ oxidase-reactive oxygen species (ROS)-connective tissue growth factor (CTGF) signaling pathway [28]. However, those results were obtained just from animals and need to be supported by clinical trials.

In our study, stratified randomization will be used to reduce the possibility of variable imbalance. Thus, no difference will exist in the use of ACEI, ARB and aldosterone antagonist between groups. Nonetheless, it is possible that some baseline levels are still not matched after randomization. In this case, we believe that it occurs "by chance" and is a small probability event. Those variables $(p<0.05)$ will be adjusted. But in addition to statistical considerations, we will also assess the relationship between the adjusted covariates and the end points, rather than unselectively adjust them.

This study has some limitations. First, though the proposed study is designed as a randomized, placebocontrolled study, it is a single-center study, which may cause the baseline drift of the selected patients. Second, the causes of $\mathrm{LVH}$ are various. Hypertension, obesity and other pathologic disorders can cause an increase in the hemodynamic burden and lead to LVH. Thus, the findings of this study may not be applied to LVH caused by all diseases.

In conclusion, this trial will first investigate trimetazidine treatment (35 mg twice a day for 12 months) on the ventricular remodeling in CAD patients with $\mathrm{LVH}$. We expect that the study results will confirm whether trimetazidine treatment can significantly improve ventricular remodeling.

\begin{abstract}
Abbreviations
CAD: Coronary artery disease; PCI: Percutaneous coronary intervention; LVH: Left ventricular hypertrophy; MACEs: Major adverse cardiovascular events; LVM: Left ventricular mass; LVMI: Left ventricular mass index; ChiCTR: Chinese clinical trial registry; ACEl: Angiotensin-converting enzyme inhibitor; ARB: Angiotensin receptor blocker; 6-MWT: 6-min walk test; CRFs: Case report forms; CBC: Complete blood count; ALT: Alanine aminotransferase; AST: Aspartate aminotransferase; Scr: Serum creatinine; BUN: Blood urea nitrogen; LDL-C: Low-density lipoprotein cholesterol; NTproBNP: N-terminal pro-B-type natriuretic peptide; IVST: Interventricular septum thickness; LVEDd: Left ventricular end-diastolic dimension; LVPWT: Left ventricular posterior wall thickness; SF-36: Short form-36; ITT: Intention-to-treat; SD: Standard deviation; SPSS: Statistical Package for Social Sciences; ATP: Adenosine triphosphate; ACS: Acute coronary syndrome; NSTEMI: Non-ST segment elevation myocardial infarction; NPY: Neuropeptide Y; NADPH: Nicotinamide adenine dinucleotide phosphate; ROS: Reactive oxygen species; CTGF: Connective tissue growth factor; ECG: Electrocardiography; UA: Uric acid; TC: Total cholesterol; HDLC: How-density lipoprotein cholesterol; TG: Triglyceride; CTnT/cTnl: Cardiac troponin-T/ cardiac troponin-l; ULN: Upper limit of normal range; eGFR: Estimated glomerular filtration rate; LVEF: Left ventricular ejection fraction
\end{abstract}

\section{Acknowledgements}

The authors would like to thank the members of data monitoring committee.

\section{Authors' contributions}

Concept - LW, WZ; Design - LW, WZ; Supervision - WZ; Fundings - LW, WZ; Materials - YiL, YaL, MW, JH, CJ; Literature search - YiL, YaL, CJ, MW, JH; Writing -YiL, YaL, LW, MW, JH, CJ; Critical review - WZ. All authors have read and approved the manuscript.

\section{Funding}

Our study protocol underwent full external peer-review during funding process which was carried out by China Cardiovascular Association (home page https://www.ccahouse.org/index.html). In line with the principle of fairness and justice, the expert jury conducted a letter review and then a total of 33 contestants were shortlisted for the interview according to the score ranking. All review experts were independent from the authors. This work was finally supported by China Cardiovascular Association-Cardiac Rehabilitation and Metabolic Therapy research fund (No. CCA-CRMT-1712), Young Talents Project of Medical and Health Research in Zhejiang Province (No. 2019RC188) and Natural Science Foundation of Zhejiang Province (LY18H020007). The funders were not involved in study design and in writing the manuscript.

\section{Availability of data and materials}

Not applicable.

\section{Ethics approval and consent to participate}

The study was approved by the Ethics Committee of Sir Run Run Shaw Hospital of Zhejiang University (KY20180508-25) and complied with the declaration of Helsinki. Written informed consent to participate will be obtained from each patient before enrollment in the study. This study has been registered in Chinese Clinical Trial Registry (http://www.chictr.org.cn; 
ChiCTR1800017876) on 19 Aug 2018. The study's recruitment is still ongoing and the recruitment time is from 2018-09-01 to 2020-12-31, which is also available on Chinese Clinical Trial Registry.

\section{Consent for publication}

Written informed consents should be obtained from patients.

\section{Competing interests}

The authors declare that they have no competing interests.

Received: 16 February 2020 Accepted: 27 May 2020

Published online: 05 June 2020

\section{References}

1. McCullough PA. Coronary artery disease. Clin J Am Soc Nephrol. 2007;2(3): 611-6.

2. Narasimhan S, McKay K, Bainey KR. Coronary artery disease in south Asians. Cardiol Rev. 2012;20(6):304-11.

3. Messerli FH, Ketelhut R. Left ventricular hypertrophy: a pressureindependent cardiovascular risk factor. J Cardiovasc Pharmacol. 1993; 22(Suppl 1):S7-13.

4. Brown DL. Effect of left ventricular hypertrophy on long-term survival of patients with coronary artery disease following percutaneous coronary intervention. Heart Int. 2009:4(1):e9.

5. Malek LA, Spiewak M, Klopotowski M, Petryka J, Mazurkiewicz L, Kruk M, et al. Influence of left ventricular hypertrophy on infarct size and left ventricular ejection fraction in ST-elevation myocardial infarction. Eur $J$ Radiol. 2012;81(3):e177-81.

6. Nepper-Christensen L, Lønborg J, Ahtarovski KA, Høfsten DE, Kyhl K, Ghotbi $A A$, et al. Left ventricular hypertrophy is associated with increased infarct size and decreased myocardial salvage in patients with ST-segment elevation myocardial infarction undergoing primary percutaneous coronary intervention. J Am Heart Assoc. 2017:6:1.

7. Shimizu I, Minamino T. Physiological and pathological cardiac hypertrophy. J Mol Cell Cardiol. 2016:97:245-62.

8. Tuomainen T, Tavi P. The role of cardiac energy metabolism in cardiac hypertrophy and failure. Exp Cell Res. 2017:360(1):12-8.

9. Cecchi F, Olivotto I, Gistri R, Lorenzoni R, Chiriatti G, Camici PG. Coronary microvascular dysfunction and prognosis in hypertrophic cardiomyopathy. N Engl J Med. 2003;349(11):1027-35.

10. Szwed H, Pachocki R, Domzal-Bochenska M, Szymczak K, Szydlowski Z, Paradowski A, et al. Efficacy and tolerance of trimetazidine, a metabolic antianginal, in combination with a hemodynamic antianginal in stable exertion angina. TRIMPOL I, a multicenter study. Presse Med. 2000;29(10): 533-8.

11. Szwed H, Sadowski Z, Elikowski W, Koronkiewicz A, Mamcarz A, Orszulak W, et al. Combination treatment in stable effort angina using trimetazidine and metoprolol: results of a randomized, double-blind, multicentre study (TRIMPOL II). TRIMetazidine in POLand. Eur Heart J. 2001;22(24):2267-74.

12. Vitale C, Spoletini I, Malorni W, Perrone-Filardi P, Volterrani M, Rosano GM. Efficacy of trimetazidine on functional capacity in symptomatic patients with stable exertional angina--the VASCO-angina study. Int J Cardiol. 2013; 168(2):1078-81.

13. Kantor PF, Lucien A, Kozak R, Lopaschuk GD. The antianginal drug trimetazidine shifts cardiac energy metabolism from fatty acid oxidation to glucose oxidation by inhibiting mitochondrial long-chain 3-ketoacyl coenzyme a thiolase. Circ Res. 2000;86(5):580-8.

14. Guarnieri C, Muscari C. Effect of trimetazidine on mitochondrial function and oxidative damage during reperfusion of ischemic hypertrophied rat myocardium. Pharmacology. 1993;46(6):324-31.

15. Cross HR. Trimetazidine: a novel protective role via maintenance of $\mathrm{Na}(+) / \mathrm{K}$ (+)-ATPase activity? Cardiovasc Res. 2000;47(4):637-9.

16. Lee L, Horowitz J, Frenneaux M. Metabolic manipulation in ischaemic heart disease, a novel approach to treatment. Eur Heart J. 2004;25(8):634-41.

17. Guarnieri $C$, Muscari C. Beneficial effects of trimetazidine on mitochondrial function and superoxide production in the cardiac muscle. Cardiovasc Drugs Ther. 1990;4(Suppl 4):814-5.

18. El Banani H, Bernard M, Baetz D, Cabanes E, Cozzone P, Lucien A, et al. Changes in intracellular sodium and $\mathrm{pH}$ during ischaemia-reperfusion are attenuated by trimetazidine. Comparison between low- and zero-flow ischaemia. Cardiovasc Res. 2000;47(4):688-96.
19. Beygui F. Trimetazidine as adjunctive therapy to primary PTCA for acute myocardial infarction. Rev Port Cardiol. 2000;19(Suppl 5):V31-4.

20. Labrou A, Giannoglou G, Zioutas D, Fragakis N, Katsaris G, Louridas G. Trimetazidine administration minimizes myocardial damage and improves left ventricular function after percutaneous coronary intervention. Am J Cardiovasc Drugs. 2007;7(2):143-50.

21. Lang RM, Badano LP, Mor-Avi V, Afilalo J, Armstrong A, Ernande L, et al. Recommendations for cardiac chamber quantification by echocardiography in adults: an update from the American Society of Echocardiography and the European Association of Cardiovascular Imaging. Eur Heart J Cardiovasc Imaging. 2015;16(3):233-70.

22. Guyatt GH, Sullivan MJ, Thompson PJ, Fallen EL, Pugsley SO, Taylor DW, et al. The 6-minute walk: a new measure of exercise capacity in patients with chronic heart failure. Can Med Assoc J. 1985;132(8):919-23.

23. Hamirani YS, Kundu BK, Zhong M, McBride A, Li Y, Davogustto GE, et al. Noninvasive detection of early metabolic left ventricular remodeling in systemic hypertension. Cardiology. 2016;133(3):157-62.

24. Ferrari R, Camici PG, Crea F, Danchin N, Fox K, Maggioni AP, et al. Expert consensus document: a 'diamond' approach to personalized treatment of angina. Nat Rev Cardiol. 2018;15(2):120-32.

25. Demirelli S, Karakelleoglu S, Gundogdu F, Tas MH, Kaya A, Duman H, et al. The impact of Trimetazidine treatment on left ventricular functions and plasma brain natriuretic peptide levels in patients with non-ST segment elevation myocardial infarction undergoing percutaneous coronary intervention. Korean Circ J. 2013;43(7):462-7.

26. Xu X, Zhang W, Zhou Y, Zhao Y, Liu Y, Shi D, et al. Effect of trimetazidine on recurrent angina pectoris and left ventricular structure in elderly multivessel coronary heart disease patients with diabetes mellitus after drug-eluting stent implantation: a single-Centre, prospective, randomized, double-blind study at 2-year follow-up. Clin Drug Investig. 2014;34(4):251-8.

27. Chen A, Li W, Chen X, Shen Y, Dai W, Dong Q, et al. Trimetazidine attenuates pressure overload-induced early cardiac energy dysfunction via regulation of neuropeptide $Y$ system in a rat model of abdominal aortic constriction. BMC Cardiovasc Disord. 2016;16(1):225.

28. Liu X, Gai Y, Liu F, Gao W, Zhang Y, Xu M, et al. Trimetazidine inhibits pressure overload-induced cardiac fibrosis through NADPH oxidase-ROSCTGF pathway. Cardiovasc Res. 2010;88(1):150-8.

\section{Publisher's Note}

Springer Nature remains neutral with regard to jurisdictional claims in published maps and institutional affiliations.

Ready to submit your research? Choose BMC and benefit from:

- fast, convenient online submission

- thorough peer review by experienced researchers in your field

- rapid publication on acceptance

- support for research data, including large and complex data types

- gold Open Access which fosters wider collaboration and increased citations

- maximum visibility for your research: over $100 \mathrm{M}$ website views per year

At $\mathrm{BMC}$, research is always in progress.

Learn more biomedcentral.com/submissions 